\title{
A Two Years Follow Up of a Surgical Treatment and Reconstruction Using Platelet Rich Fibrin for Central Giant Cell Granuloma of The Mandible _ A Case Report
} Tarek kasem ${ }^{1}$, Salma Albati ${ }^{2}$, Jihad Alokaily ${ }^{3}$, Ali Alsalim ${ }^{4}$

Dental Department, AlRass general hospital, Qassim, Kingdom of Saudi Arabia.

\begin{abstract}
Background: Central giant cell granuloma (CGCG) is a benign aggressive destructive osteolytic lesion of osteoclastic origin. The central giant cell granuloma is often found in the mandible, anterior to the first molars. It most commonly occurs in patients under the age of 30 , with a clear female prevalence.

Objectives: The aim of this report was to present a case of CGCG of the mandible and to describe the effect of platelet rich fibrin along with the surgical treatment on such aggressive lesions.

Patients and methods: A 29 years old man referred from Hafer Albaten General Hospital to our Department of Oral and Maxillofacial Surgery with a chief complaint of painless swelling on the left lower back jaw region for 6 months. An unusual treatment modality had been carried out in Department of Oral and Maxillofacial Surgery at Alrass General Hospital. Aggressive curettage along with peripheral osteotomy has been done followed by reconstruction with Platelet rich fibrin $(P R F)$ as an autologous grafting material.
\end{abstract}

Results: A Three months, six months, One year and Two years follow up revealed a good healing of the osseous lesion with no signs of recurrence.

Conclusion: Curettage of the tumor mass, followed by the removal of the peripheral bony margins results in a low recurrence rate and good prognosis.

Keywords: Central Giant Cell Granuloma, Platelet rich fibrin, Autologous graft, Reconstructive surgery.

\section{INTRODUCTION}

Central giant cell granuloma (CGCG) is described as a benign lesion affecting the mandible and maxilla that consists of a massive fibrohistiocytic proliferation with numerous heavily hemosidrinladen multinucleated giant cells. CGCG was first described by Jaffe in 1953. It is an uncommon, benign and proliferative nonneoplastic process. Jaffe considered it as a locally reparative reaction of bone, which can be possibly due to either an inflammatory response, hemorrhage or local trauma. Females are affected more frequently than males. Most lesions occur in the molar and premolar area, some of these extending up to the ascending ramus (1).

\section{AIM OF THE STUDY}

The purpose of this clinical report was to describe the effect of platelet rich fibrin along with the surgical treatment on such aggressive lesions.

\section{CASE REPORT}

A 29 year - old man referred from Hafer Albaten General Hospital to our Department of Oral and Maxillofacial Surgery with a chief complaint of painless swelling on the left lower back jaw region since 6 months.

On examination, a swelling on the left side was revealed on posterior mandibular region, which was firm and painless. The patient had difficulty in speech and chewing, as there was a limited mouth opening. Examination revealed large swelling, focal, non tender, non-fluctuant and non compressible, restricting the mandibular movements. On extra-oral examination, a single, focal, swelling was seen on the left side of the mandible. The swelling measured about $4 \mathrm{~cm} \times 4 \mathrm{~cm}$. The surface of the swelling was smooth and extended along the lower border of the mandible (figure 1A \& 1B). Intra oral examination revealed a solitary swelling in the left lower buccal vestibule in the third molar region extending posteriorly to the posterior border of mandible.

Radiographically Panoramic X-ray showed a multilocular, radiolucent destructive lesion in the mandibular left third molar region (Figure 2). 3D CTScan showed a multiloculated lesion with areas of bone destruction and erosion extending to mandibular ramus (Figure 3).

Based on the clinical and radiographic examination, a provisional diagnosis of Ameloblastoma, True giant cell lesion, Odontogenic keratocyst, Hemangioma, Aneurysmal bone cyst, Traumatic bone cyst was made.

Fine-needle aspiration cytology was performed which came out to be negative to confirm the diagnosis. An incisional biopsy was planned. Biopsied specimen revealed connective tissue made up of mature collagen fibers, fibroblasts and numerous multinucleate giant cells with foci of osseous structures. On the basis of histopathologic and radiological findings, a diagnosis of aggressive CGCG was established (Figure 4).

Pre-operative evaluation was performed before approaching the patient under local anesthesia. Routine hemogram was done, which turned out to be normal.

\section{Procedure:}

The tumor mass was removed through an intraoral approach and curettage with peripheral ostectomy with preservation of the continuity of the mandible was performed (Figures 5A \& 5B). Tooth \#38 was extracted. The inferior alveolar nerve was preserved. Immediate reconstruction with platelet rich 
fibrin and suturing of the surgical site was done (Figure 6).

\section{DISCUSSION}

CGCG was hypothesized that the lesion is not a true neoplasm but merely the result of a local reparative reaction. CGCG appears as a painless expansile mass. The clinical behavior of the CGCG ranges from a slowly growing asymptomatic swelling to an aggressive lesion that manifests with pain, local destruction of bone, root resorption, or displacement of teeth ${ }^{(2)}$.

Giant cells are the most prominent histopathologic feature of CGCG and many investigative studies have been directed to the role of the original mononuclear cells in its pathogenesis. The mononuclear cells can form osteoclasts like giant cells in vitro by the development of osteolytic lesions. Besides osteoclasts, the mononuclear cells differentiate themselves in macrophages that play a critical in connective tissue during inflammatory and reparative process ${ }^{(3)}$.

The radiologic features of giant cell granuloma have not been clearly defined; the lesion may appear as unilocular or multilocular radiolucency with welldefined or ill-defined margins with varying degrees of expansion of the cortical plates. Radiographic appearance of the lesion is not pathognomic and may be confused with that of many other lesion of the jaws ${ }^{(4)}$.

CGCG occurs predominantly in children or young adults, with approximately $75 \%$ of cases presenting before 30 years of age. However, it really can occur at any age. Females are affected more frequently than males, with a ratio of $2: 1$ and more than $70 \%$ of CGCGs occur in the mandible and less than $30 \%$ in the maxilla with a preference for the anterior portions of both bones (5)

The treatment of CGCG of the jaws is performed according to the following factors: aggressive versus non-aggressive behavior, location, size and radiographic appearance. Surgical options range from large (en bloc resection) to more conservative approaches (curettage). The traditional therapy of CGCG is local curettage (this has been associated with a high success rate-80\%), peripheral osteotomy and excision if needed [6,7]. Surgical treatment of CGCGs can be associated with recurrence and serious facial mutilation and loss of teeth and tooth germs. To avoid such disadvantages, a number of alternative nonsurgical therapies including interferon alpha-2a, calcitonin and intralesional corticosteroid injection have been advocated for the management of CGCG. Non-surgical treatment of CGCG is probably a good treatment option for small slowly enlarging lesions. Successful treatment of painful, large, and rapidly growing lesions is more likely achieved by surgical removal. In the literature, recurrence rates vary between $11 \%$ and $35 \%{ }^{[6]}$.

In the present case of CGCG, there was a multilocular, radiolucent expansile lesion, which involved the cortical bone. Surgical curettage has been done followed by peripheral osteotomy and immediate reconstruction with platelet-rich fibrin. The effect of using platelet-rich fibrin in combination with the surgical intervention has been detected from the first 3 months of recall. As there was a remarkable regression of the lesion and regeneration of the osseous defect. Then a one year follow up revealed a complete healing of the lesion with no signs of recurrence.

\section{CONCLUSION}

The most widely accepted method of surgical treatment of CGCG is aggressive curettage. Curettage of the tumor mass, followed by the removal of the peripheral bony margins results in a low recurrence rate and good prognosis. If soft tissues and periosteum are preserved, and only the bony component is excised, then it is possible to reconstruct the surgical defect with autologous grafts (platelet-rich fibrin). By doing this bone continuity is maintained and prosthetic rehabilitation can be safely performed.

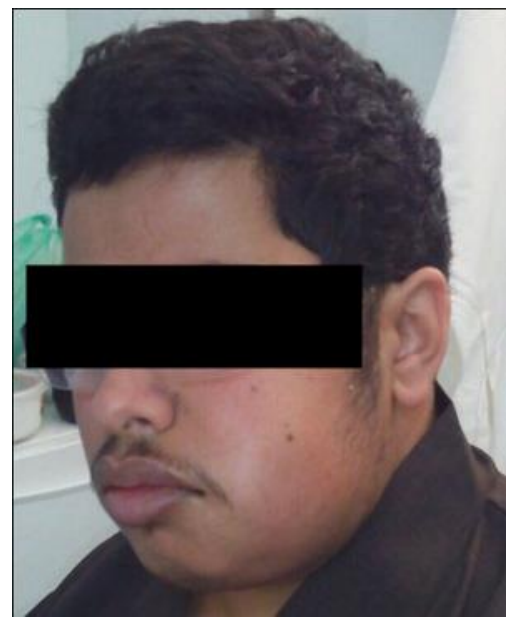

Figure (1A)

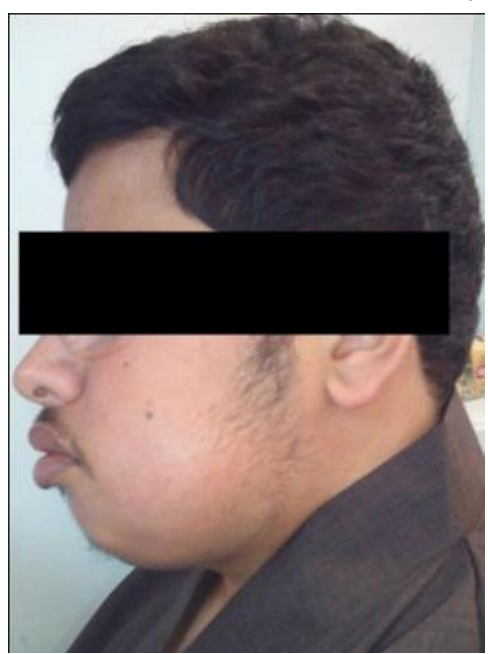

Figure (1B)

Figure (1A,1B): Swelling was seen on the left side of the mandible measuring about $4 \mathrm{~cm} \times 4 \mathrm{~cm}$. 


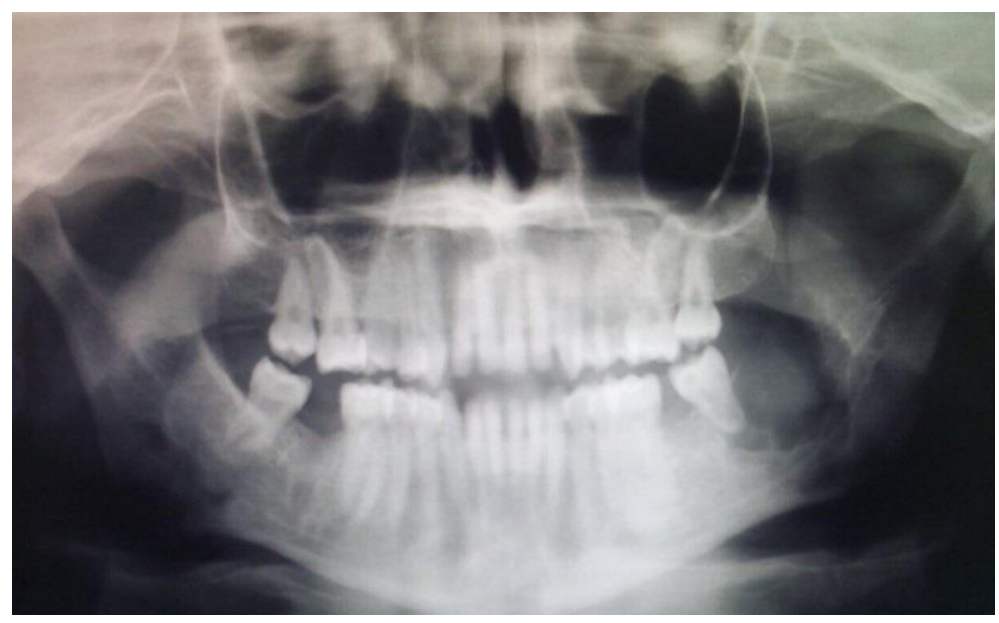

Figure (2): Panoramic X-ray showed a multilocular, radiolucent destructive lesion in the mandibular left third molar region

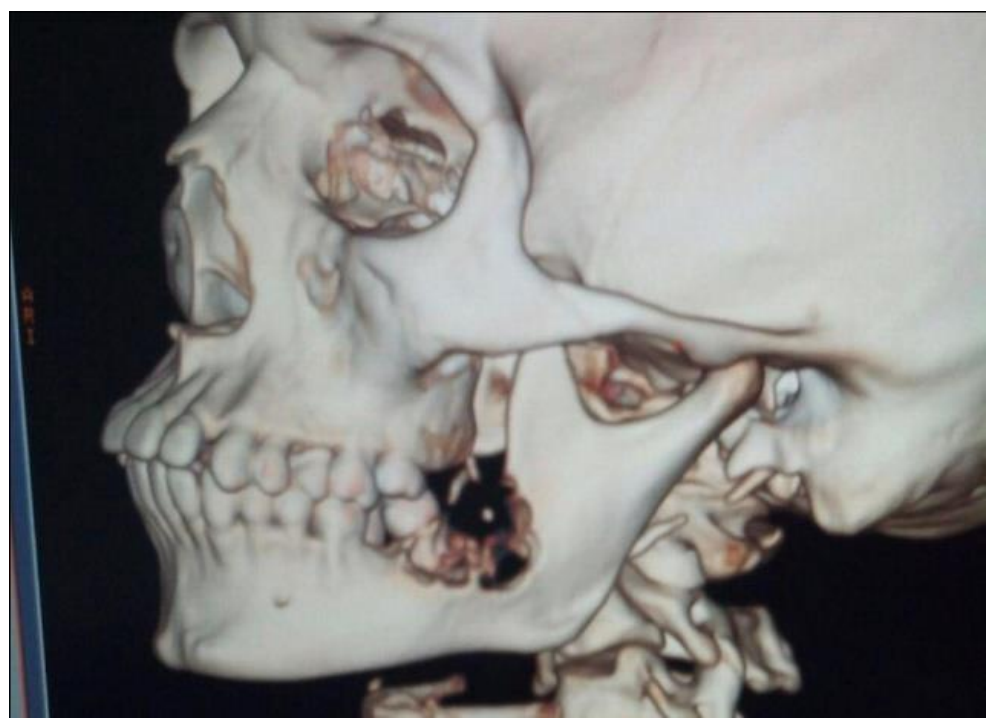

Figure (3): 3D CT-Scan showed a multiloculated lesion with areas of bone destruction and erosion extending to mandibular ramus

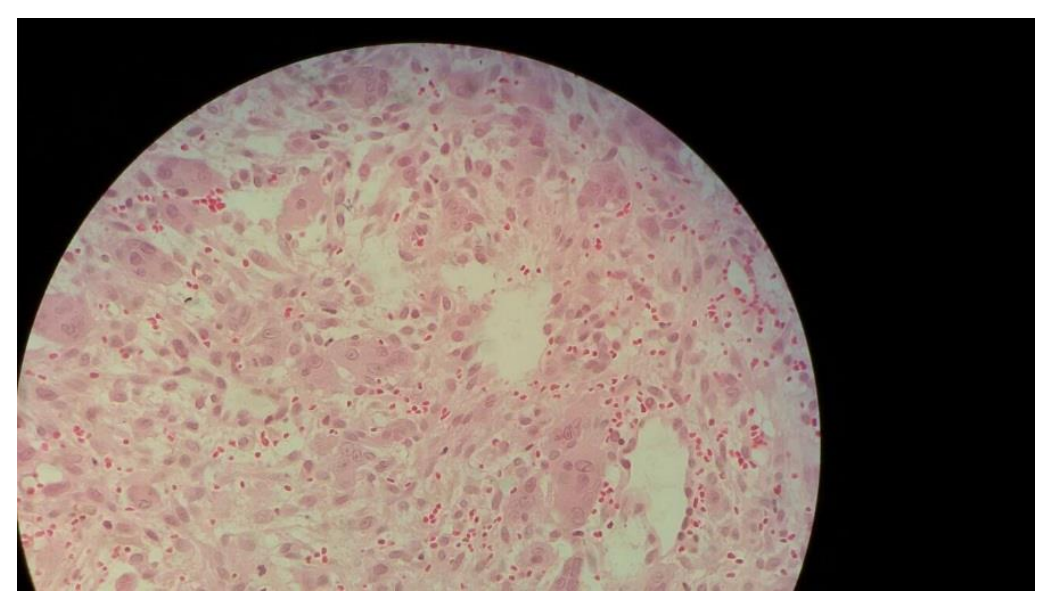

Figure (4): Biopsied specimen revealed connective tissue made up of mature collagen fibers, fibroblasts and numerous multinucleate giant cells with foci of osseous structures. 


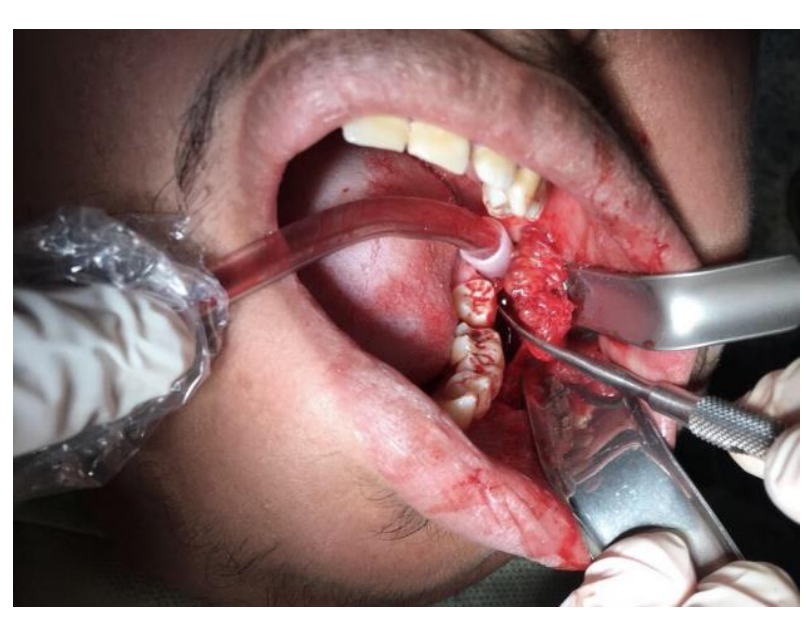

Figure (5A)

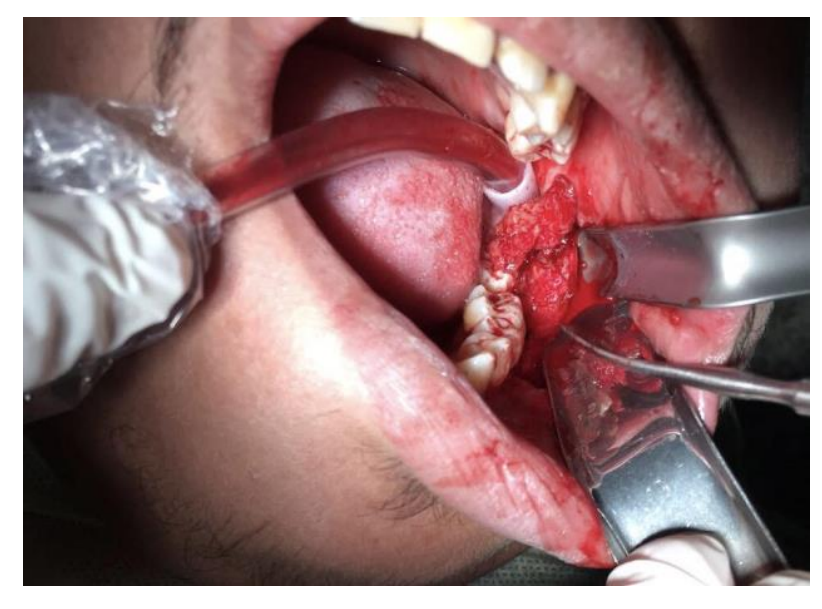

Figure (5B)

Figure (5A,5B): Surgical removal of the mass.
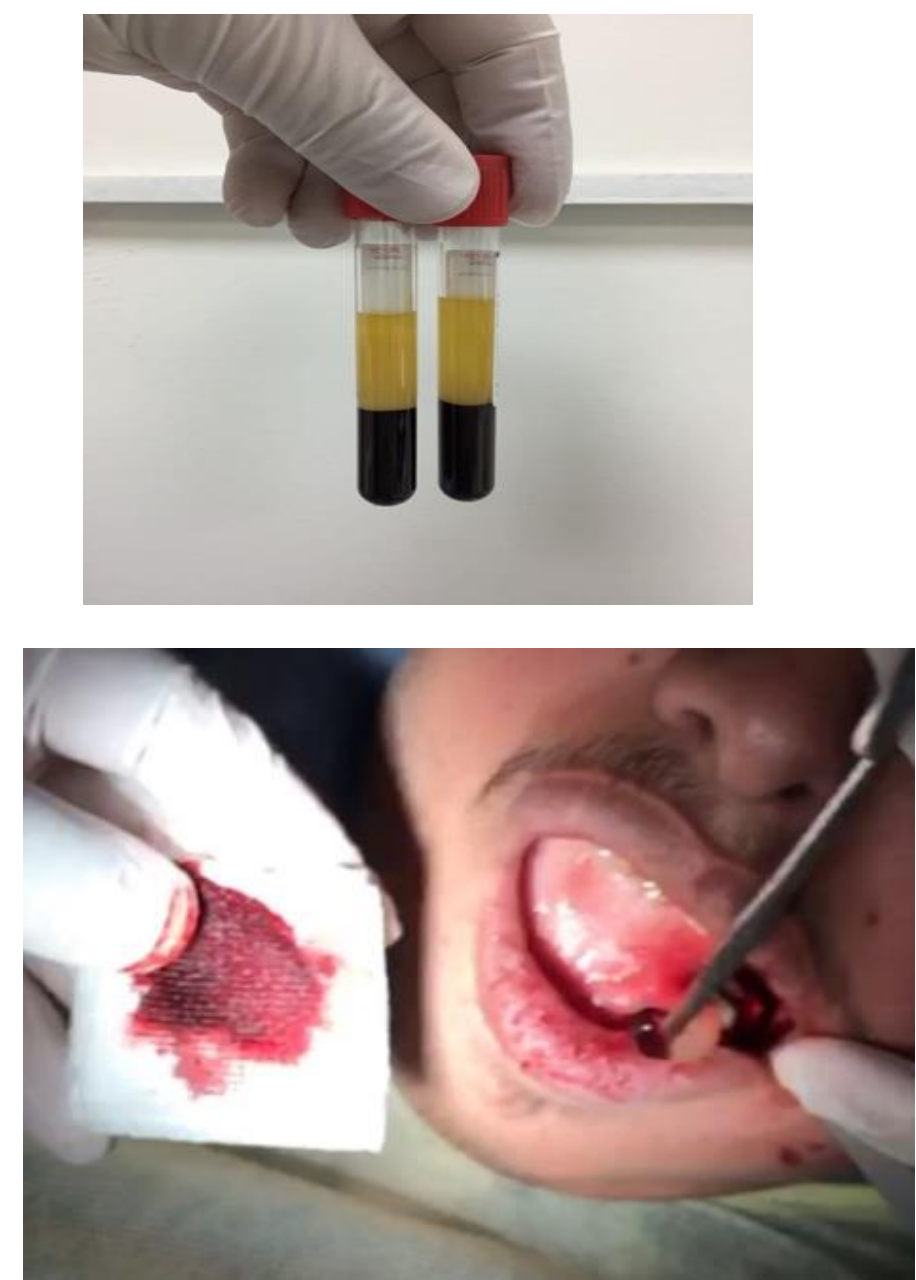

Figure (6): Immediate reconstruction with platelet rich fibrin was done and suturing. 
ejhm.journals.ekb.eg
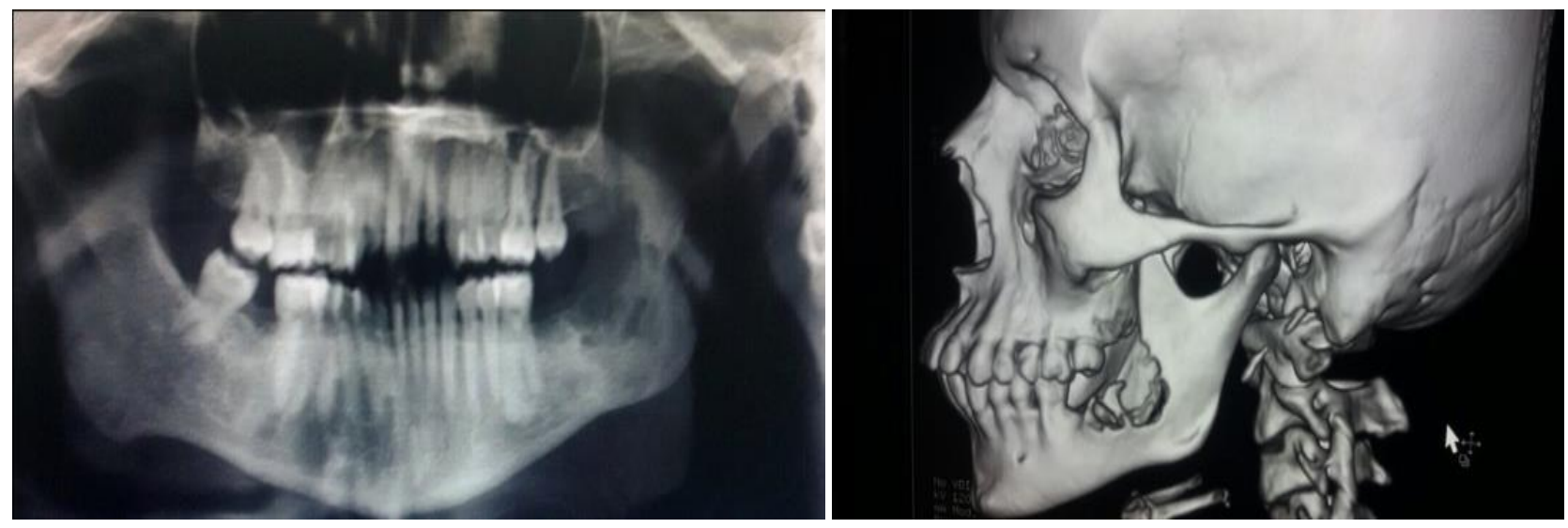

Figure (7): 3 months follow up

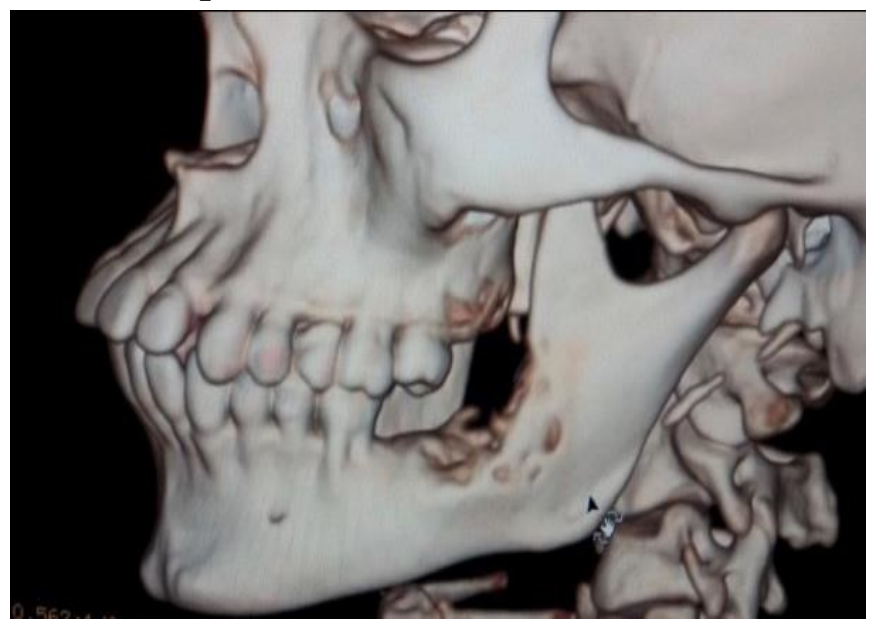

Figure (8): 6 months follow up

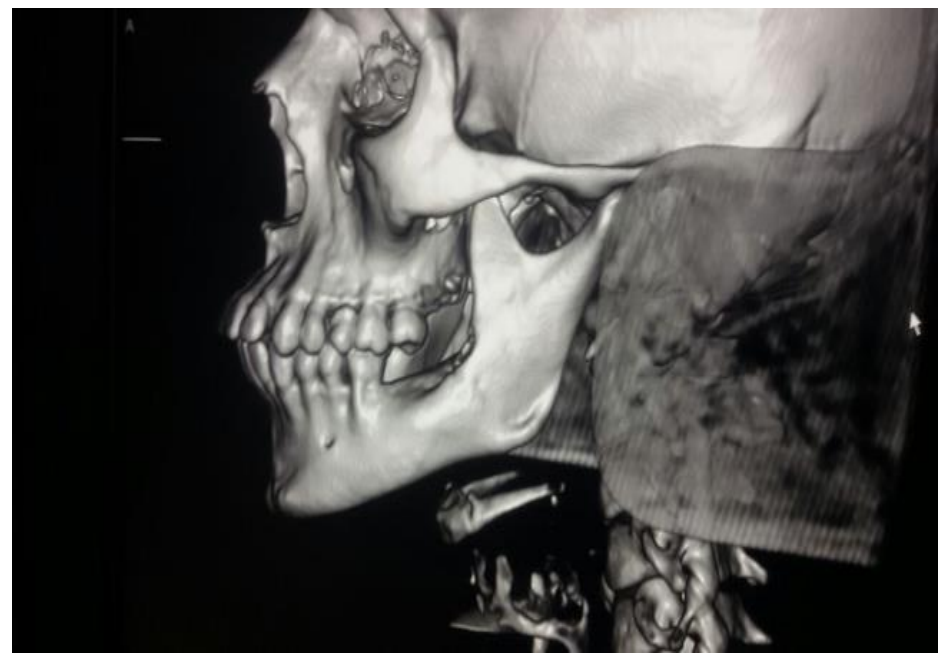

Figure (9): one year follow up 


\section{REFERENCES}

1. Jaffe HL (1953): Giant-cell reparative granuloma, traumatic bone cyst, and fibrous (fi bro-oseous) dysplasia of the jaw bones. Oral Surg Oral Med Oral Pathol., 6:15975.

2. Austin LT, Dahlin CD, Royer QR(1955):Central giant cell granuloma and related condition affecting the jaw bone. Oral Surg Oral Med Oral Pathol .,12:1259.

3. Whitaker SB, Waldron CA (19932): Central giant cell lesions of the jaws. A clinical, radiologic, and histopathologic study. Oral Surg OralMed Oral Pathol., 75: 199-208.

4. Chuong R, Kaban LB, Kozakewich H, Perez-Atyade A(1986):Central giant cell lesions of the jaws. A clinicopathologic study. J Oral Maxillofac Surg .,44:70813.

5. Shafer W G, Hine mk, Levy BM (1983): A text book of oral pathology $4^{\text {th }}$ ed. Philadelphia; WB Saunders, Pp: 260265.

6. Rachmiel A, Emodi O, Sabo E, Aizenbud D, Peled M (2012): Combined treatment of aggressive central giant cell granuloma in the lower jaw. J Cranio-maxillofac Surg., 40 (3): 292-7.

7. De Lange J, van den Akker HP, van den Berg $\mathbf{H}(\mathbf{2 0 0 7 )}$ :Central giant cell granuloma of the jaw: a review of the literature with emphasis on therapy options. Oral Surg Oral Med Oral Pathol Oral Radiol Endod., 104(5): 603-615. 